\title{
Bindehautinfektionen
}

\author{
P. Steven, L. M. Heindl \\ Zentrum für Augenheilkunde, Uniklinik Köln
}

Infektionen der Bindehaut gehören zu den häufigsten Augenerkrankungen weltweit. Hierbei reicht das Spektrum der Erreger von Bakterien über Viren und Pilzen bis hin zu Parasiten. Regionale Unterschiede beeinflussen die Krankheitshäufigkeit, wobei zum Beispiel parasitäre Erkrankungen oder Pilzkonjunktivitiden in mitteleuropäischen Breiten eher selten sind.
Im diesem Beitrag werden die unterschiedlichen Erregergruppen systematisch beschrieben zusammen mit der jeweiligen Symptomatik, Diagnostik sowie der Behandlung und Prognose.

\section{Bakterielle Konjunktivitis}

\section{Zusammenfassung}

Bakterielle Infektionen der Konjunktiven sind häufig und werden oft von Nicht-Augenärzten behandelt. Vor allem bei Kindern tritt eine Infektion sehr häufig auf und wird daher primär durch den Pädiater mittels antibiotischer Augentropfen therapiert.

\section{Epidemiologie und Ätiologie}

Epidemiologie. Für Deutschland gibt es keine epidemiologischen Daten in Bezug auf die Häufigkeit bakterieller Bindehautentzündungen. Für die USA wurden für das Jahr 2005 jedoch ca. 4 Mio. Behandlungsfälle geschätzt, wobei nahezu ein Viertel auf Kinder im Alter zwischen 0 und 2 Jahre entfielen, knapp 30\% auf Kinder im Alter von 3-9 Jahre und knapp 15\% auf Kinder und Jugendliche bis 19 Jahre [1].

Nur 35\% der Erkrankungen betreffen Erwachsene. Daher erfolgt die Behandlung der bakteriellen Konjunktivitis oft durch Kinder- und Allgemeinärzte mittels antibiotischer Augentropfen.

Ätiologie. Die Augenoberfläche verfügt über ein äußerst wirksames Abwehrsystem, dass sich aus Komponenten des angeborenen und des erworbenen Immunsystems zusammensetzt. So können zum Beispiel Muzine und antimikrobielle Peptide des Tränenfilms

$\begin{array}{ll}\text { Abkürzungen } \\ \text { HSV } & \text { Herpes-simplex-Virus } \\ \text { KCE } & \text { Keratoconjunctivitis epidemica } \\ \text { MRSA } & \text { methicillinresistenter Staphylococcus aureus } \\ \text { PCR } & \begin{array}{l}\text { Polymerase Chain Reaction/Polymerase- } \\ \text { Kettenreaktion }\end{array} \\ \text { PKF } & \text { pharyngokonjunktivales Fieber }\end{array}$

und des Bindehautepithels direkt bakteriostatische Effekte erzielen. Sezernierte Immunglobuline unterstützen diese oberflächenlokalisierte Abwehr. Sollten Erreger die Schleimhautbarriere durchbrechen, ist das diffuse und organisierte Konjunktiva-assoziierte lymphatische Gewebe als weitere funktionelle Barriere an der Erregerabwehr beteiligt.

Aufgrund dieses hochwirksamen Immunsystems ist die sog. kommensale Flora der Augenoberfläche, die vor allem aus Staphylococcus epidermidis, Diphtheroiden Stäbchen und Propionibacterium acnes besteht, für das Auge meistens gefahrlos.

Unter bestimmten Bedingungen können jedoch diese an sich harmlosen Bakterien oder andere Pathogene die Schleimhautbarriere erleichtert durchbrechen. Risikofaktoren für eine bakterielle Konjuntivitis s. Checkliste. 


\section{Checkliste}

Risikofaktoren für eine bakterielle Konjunktivitis

- trockenes Auge

- Bindehautvernarbungen (vernarbendes Schleimhautpemphigoid, Stevens-Johnson-Syndrom)

- okuläre Graft-versus-Host-Erkrankung

- reduzierte Lidschlagfrequenz

- Lagophthalmus

- Exophthalmus

- immunsuppressive Therapie (z. B. topische oder systemische Steroide)

- immunsupprimierende Erkrankungen (z.B. Malignome)

- Mangelernährung

- Tränenwegsverschlüsse

- operative Eingriffe an der Augenoberfläche

- Bestrahlung

- Trauma
Streptococcus pneumoniae und Haemophilus influenzae sind typische Erreger von bakteriellen Konjunktivitiden bei Kindern.

Bei der Neugeborenenkonjunktivitis (Ophthalmia neonatorum) spielen vor allem sexuell übertragbare Erreger wie Neisseria gonorrhoeae oder Chlamydia trachomatis (neben Herpesviren) eine Rolle.

In den vergangenen Jahren wurde eine Zunahme an MRSA-Infektionen der Bindehaut beobachtet. Insbesondere bei Patienten aus Pflegeeinrichtungen, aber auch zunehmend in der restlichen Bevölkerung, werden Abstrichnachweise erbracht. Bei Staphylococcusaureus-positiven Abstrichen liegt die Inzidenz von MRSA zwischen 17-30\%.

Als besondere Erreger gelten Chlamydien, da sie nur mittels spezieller Verfahren nachgewiesen werden können. Bedeutsam sind in nordeuropäischen Regionen vor allem Chlamydien der Spezies Chlamydia trachomatis Serovar D-K, die eine EinschlusskörperchenKonjunktivitis bei Neugeborenen und Erwachsenen auslösen können.

Weltweit kommt es jährlich zu 89 Mio. Neuerkrankungen mit Chlamydien, vor allem in Entwicklungsländern. Ein Großteil der Infektionen, das sog. Trachom, wird durch die Serovare A-C verursacht; ergänzend verursachen die Serovare L1-3 ein Lymphogranuloma venerum.

Das Trachom gehört weiterhin nach der Katarakt zu den weltweit häufigsten Erblindungsursachen, betrifft sehr häufig Kinder und ist zumeist mit einem Mangel an Wasser, niedrigem sozioökonomischen Status und schlechter Hygiene assoziiert.

Das Trachom wird in 5 Stadien eingeteilt:

1. Follikuläre Konjunktivitis mit mindestens 5 Follikeln $>0,5 \mathrm{~mm}$ in der oberen tarsalen Konjunktiva.

2. Starke trachomassoziierte Konjunktivitis mit Verdickung der tarsalen Konjunktiva.

3. Vernarbende Konjunktivitis der tarsalen Konjunktiva.

4. Trichiasis, wobei mindestens eine Wimper den Augapfel berührt.

5. Korneale Trübung. 


\section{Überblick}

Stadieneinteilung des Trachoms

1. follikuläre Konjunktivitis mit mindestens 5 Follikeln $>0,5 \mathrm{~mm}$ in der oberen tarsalen Konjunktiva

2. starke trachomassoziierte Konjunktivitis mit Verdickung der tarsalen Konjunktiva

3. Vernarbende Konjunktivitis der tarsalen Konjunktiva

4. Trichiasis, wobei mindestens eine Wimper den Augapfel berührt

5. korneale Trübung

\section{Checkliste}

Übertragungswege bakterieller Erreger

- Hände

- traumatische Fremdkörper

- Kontaktlinsen

- kontaminierte Augentropfen und -salben

- über die angrenzende Gesichtshaut/über das Augenlid

- iatrogen: okuläre oder periokuläre Eingriffe

- selten: systemische Übertragung über den Blutkreislauf

Inkubations- und Transmissionszeit schwanken abhängig vom Erreger, sie liegen aber im Mittel bei ca. 1 Woche.

\section{Symptomatik}

Bezüglich Symptomatik und Verlauf werden bakterielle Konjunktivitiden in hyperakute, akute und chronische Formen eingeteilt. Alle Formen beginnen zunächst in der Frühphase mit einer serösen Sekretion, die dann durch Zunahme von sezernierten Muzinen in eine mukoide und schließlich in eine eitrige Sekretion übergeht (Abb. 1).

Die eitrige Komponente beruht dabei auf einer massiven Anflutung von Granulozyten in die Bindehaut mit folgendem Durchtritt durch das Bindehautepithel auf die Augenoberfläche. Das eitrige Sekret besteht aus Mukus, Granulozyten und Erregern und zudem aus Zelldetritus abgestorbener Epithelzellen und anderen Immunzellen wie Makrophagen und Lymphozyten.

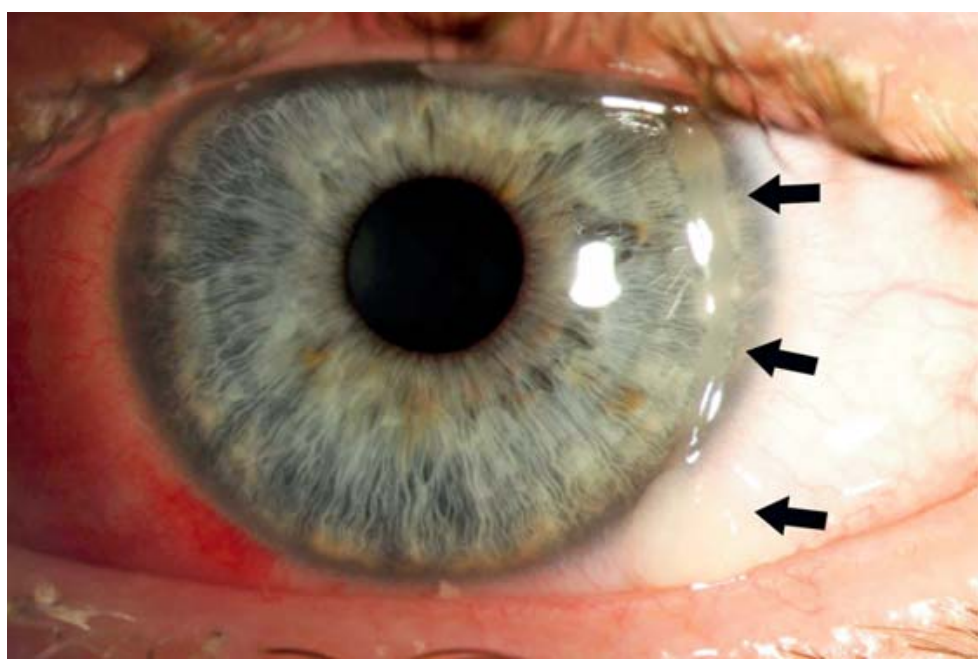

Abb. 1 Akute bakterielle Konjunktivitis mit mukopurulenter Sekretion (Pfeile).

Übertragungswege. Die bakteriellen Erreger gelangen über die unterschiedlichsten Wege auf die Augenoberfläche, wobei vor allem die Übertragung über Finger, durch traumatische Fremdkörper und mittels Kontaktlinsen zahlenmäßig von großer Bedeutung sind.

Eine Übertragung kann außerdem durch kontaminierte Augentropfen und -salben, über die angrenzende Gesichtshaut, das Augenlid oder iatrogen durch okuläre oder periokuläre Eingriffe erfolgen. In seltenen Fällen ist auch eine systemische Übertragung über den Blutkreislauf möglich. 


\section{Checkliste}

Typische klinische Zeichen einer bakteriellen Konjunktivitis sind

- seröse, mukopurulente oder purulente Sekretion

- gerötete und ödematöse Lider und Lidkanten

- verklebte Wimpern

- gerötete Konjunktiven

- Chemosis

- Bindehautfollikel

- Papillen

- Membranen und Pseudomembranen

Weitere klinische Zeichen sind gerötete und ödematöse Lider und Lidkanten, verklebte Wimpern, gerötete Konjunktiven, Chemosis, Bindehautfollikel und Papillen. Weiterhin können Membranen und Pseudomembranen auftreten.

Echte Membranen sind mit dem darunterliegenden Epithel verbunden, sodass eine Entfernung zu einer blutenden Wunde führt. Pseudomembranen hingegen können ohne Epithelschaden entfernt werden [3].

Hyperakute Form. Die hyperakute Form ist insgesamt eher selten und durch ein sehr schnelles Auftreten, massive eitrige Sekretion und starke Schmerzen charakterisiert. Typische Erreger sind Neisseria gonorrhoeae oder Neisseria meningitidis. Hier ist ein Abstrich und ein unverzüglicher Therapiebeginn immer obligat (s. auch Diagnostik).

Die Gonokokken-Konjunktivitis bei Neugeborenen (Gonoblenorrhoe) tritt aufgrund einer obligaten Gonokokkenuntersuchung der Mutter im Rahmen der Schwangerschaftsvorsorge in Deutschland eher selten auf.

Akute Form. Die akute Form zeigt eine weniger dramatische Klinik, mit Augenrötung, mukopurulentem Sekret und moderaten Schmerzen. Es können ein oder beide Augen betroffen sein.

Chronische Form. Von einer chronischen bakteriellen Konjunktivitis spricht man bei einer Dauer von über 4 Wochen oder ständigen Rezidiven mit sehr kurzen reizfreien Intervallen. Das bakterielle Erregerspektrum reicht von Staphylokokken über tränenwegslokalisierte Actimomyceten bis hin zu Chlamydien.

\section{Diagnostik}

Typische klinische Zeichen einer bakteriellen Konjunktivitis sind wie oben schon beschrieben: seröses, mukopurulentes oder purulentes Sekret, (Blepharo)Konjunktivitis mit verklebten Wimpern, Papillen, Follikel, Pseudomembranen oder Membranen und Schmerzen. Zum Teil ist eine korneale Beteiligung vorhanden; hier wird auf den entsprechende Beitrag zu infektiösen Hornhauterkrankungen verwiesen.

Konjunktivale Zeichen wie Papillen oder Follikel sind primär unspezifische Zeichen, die nur bedingt bei der Diagnostik helfen. Beide kommen bei diversen entzündlichen Erkrankungen der Bindehaut (trockenes Auge, okuläre Allergie, bakterielle und virale Infektionen sowie als toxische Reaktion, z. B. gegen Augentropfen) vor und sind daher nicht pathognomonisch für einzelne Erkrankungen.

Zur Diagnosesicherung ist daher nur ein Erregernachweis geeignet, der mit einer mikrobiologischen Untersuchung eines Bindehautabstrichs durchgeführt wird. Da diese Abstriche bei bis zu 65\% der Fälle negativ ausfallen und gewöhnliche bakterielle Konjunktivitiden eine hohe Spontanheilungsrate aufweisen, sind Abstriche nicht bei jedem Patienten notwendig.

Bei bestimmten Patienten, z. B. bei Neugeborenen und immunsupprimierten Patienten, wird jedoch ein Abstrich immer empfohlen (s.Zielgerichtete Diagnostik). Dabei ist darauf zu achten, neben klassischen Bakterien auch an Erreger zu denken, die mit Spezialuntersuchungen (z. B. PCR bei Chlamydien) nachgewiesen werden müssen. Zudem sollte immer eine Resistenzbestimmung erfolgen.

Die Gewinnung von Material für eine Chlamydien-PCR muss dabei nicht mehr mit einem z.T. blutigen Bürstenabstrich zur Gewinnung von Epithelzellen erfolgen, sondern kann mittlerweile mit einem speziellen Abstrichtupfer und Medium auch atraumatisch-oberflächlich erfolgen [4]. 


\section{Zielgerichtete Diagnostik}

Ein Bindehautabstrich ist sinnvoll bei

- Neugeborenen

- immunsupprimierten Patienten

- Patienten mit hyperakuter Konjunktivitis

- Patienten mit chronischer Konjunktivitis

- Patienten mit follikulärer Konjunktivitis (ChlamydienPCR)

- Patienten aus Pflegeheimen oder bei bekannter MRSA-Infektion

\section{Therapie}

Die Therapie der bakteriellen Konjunktivitis findet in den meisten Fällen ohne genaue Kenntnis des verursachenden Erregers statt. In der Regel wird ein Breitspektrum-Antibiotikum topisch in Form von Augentropfen oder Augensalben appliziert. Obwohl eine bakterielle Konjunktivitis zumeist spontan in 5 Tagen abheilt, konnte in diversen Studien gezeigt werden, dass eine bakterielle Therapie zu einer schnelleren klinischen Befundbesserung und zu einer früheren Ausheilung führt und daher standardmäßig erfolgen sollte.

Die Wahl des Antibiotikums richtet sich nach verschiedenen Kriterien wie Patientensicherheit, Erregerverdacht, Preis und Nebenwirkungen. Grundsätzlich sind Breitspektrum-Antibiotika bei Therapiebeginn ohne Erregernachweis zu bevorzugen (Tab. 1).

Man unterscheidet insgesamt bakteriostatische von bakteriziden Antibiotika. Bei den bakteriostatischen Antibiotika sind das Azithromycin, Chloramphenicol und Trimethoprim zu nennen. Azithromycin wird 2-mal pro Tag über 3 Tage gegeben und ist daher sehr anwenderfreundlich, außerdem für Kinder ab dem 2. Lebensjahr zugelassen. Trimethoprim wird oft mit dem bakterioziden Antibiotikum Polymyxin B appliziert um die Wirksamkeit von Trimethoprim gegen

\section{Tabelle 1}

Antibiotikatherapie bei unkomplizierter akuter Konjunktivitis.

\begin{tabular}{|l|l|}
\hline Neugeborene & Moxifloxacin \\
\hline Kinder ab dem 2. Lebensjahr & Azithromycin \\
\hline Erwachsene & $\begin{array}{l}\text { Gentamicin, Tobramycin } \\
\text { oder Azithromycin }\end{array}$ \\
\hline
\end{tabular}

Staph. aureus mit der von Polymyxin B gegen gramnegative Erreger zu kombinieren. Aminoglykoside (Gentamicin, Tobramycin, Neomycin) wirken gegen gramnegative Erreger (Haemophilus, Enterobacter, Klebsiella, Proteus, Serratia, etc.) und insbesondere gegen Pseudomonas (nicht Neomycin) und Staphylokokken. Fluorchinolone (Ofloxacin, Levofloxacin, Moxifloxacin) weisen eine gute Wirksamkeit gegenüber Staphylokokken und Streptokokken auf.

Bei vorliegendem Erregernachweis (mit Resistenzbestimmung) sollte immer eine Therapieüberprüfung und ggf. Anpassung erfolgen.

Chlamydieninfektionen. Bei Vorliegen einer Chlamydieninfektion ist immer an einen sexuellen Übertragungsweg zu denken.

Cave. Sexualpartner müssen bei einer Chlamydieninfektion immer mitbehandelt werden, um Folgeschäden und eine Re-Infektion (sog. Ping-PongEffekt) zu vermeiden.

Zusätzlich sollte eine Vorstellung beim Dermatologen, Venerologen oder Gynäkologen empfohlen werden.

Die Therapie der Chlamydieninfektion erfolgt standardmäßig mit 500 mg Azythromycin pro Tag oral über 3 aufeinander folgende Tage. Eine topische Applikation ist bei gleichzeitiger systemischer Therapie nicht notwendig.

Alternativ ist auch die Applikation von Povidon-Jod denkbar. Hier wurde in einer Studie mit über 450 Kindern und Jugendlichen eine Wirksamkeit in der Behandlung von bakteriellen (inkl. Chlamydien-) Konjunktivitiden gezeigt, wobei die Applikation von topischem Povidon-Jod gleich wirksam wie ein topisches Breitspektrum-Antibiotikum war [6].

\section{Virale Konjunktivitis}

\section{Zusammenfassung}

Virale Bindehautentzündungen können klinisch unterschiedlich verlaufen. Eine follikuläre Konjunktivitis ist ein typisches, jedoch nicht pathognomonisches Symptom. Therapeutische Optionen umfassen vor allem antivirale Substanzen. Vor allem Infektionen mit DNA-Viren können potenziell visusbedrohliche Verläufe nehmen, daher ist eine korrekte Diagnose und Therapie von großer Bedeutung. 


\section{Tabelle 2}

Erreger bei viralen Bindehautentzündungen.

\begin{tabular}{|l|l|}
\hline DNA-Viren & RNA-Viren \\
\hline Adenoviren & Picorna-Virus \\
\hline Herpes-simplex-Virus (HSV) & Paramyxovirus \\
\hline Varizella-zoster-Virus (VZV) & Tagavirus \\
\hline Epstein-Barr-Virus (EBV) & Flavivirus \\
\hline
\end{tabular}

Zytomegalievirus (CMV)

Molluscum contagiosum (Poxvirus)

\section{Ätiologie}

Virale Bindehautentzündungen zeigen eine große Variabilität in ihrer klinischen Ausprägung. Als Erreger kommen DNA- und RNA-Viren vor (Tab. 2). Aufgrund der klinischen Relevanz werden hier vor allem Infektionen mit Adenoviren, Herpesviren und dem Molluscum-contagiosum-Virus behandelt.

Cave. Eine Beteiligung der Hornhaut bei Infektionen mit Adenoviren, HSV, VZV und CMV ist potenziell visusbedrohend und sollte daher schnell behandelt werden.

Zu den häufigsten Erregern einer viralen Konjunktivitis zählen die Adenoviren, von denen bislang 51 verschiedene humanpathogene Formen beschrieben wurden. Adenoviren verursachen unterschiedliche Erkrankungen:

- pharyngokonjunktivales Fieber (PKF)

- Keratoconjunctivitis epidemica (KCE)

- akute nicht spezifische follikuläre Konjunktivitis

- chronische Keratokonjunktivitis.

\section{Überblick}

Durch Adenoviren verursachte Bindehauterkrankungen

- pharyngokonjunktivales Fieber (PKF)

- Keratoconjunctivitis epidemica (KCE)

- akute nicht spezifische follikuläre Konjunktivitis

- chronische Keratokonjunktivitis
Die KCE ist eine hochinfektiöse Erkrankung, die über Tröpfchen übertragen wird und als Infektionsweg die Schleimhäute von Auge, Nase und Rachen nimmt. Typischerweise verläuft die Übertragung über Hände, Taschentücher, Handtücher, Türgriffe oder mangelhaft desinfizierte ophthalmologische Instrumente. Die virale Inkubationszeit beträgt 2-12 Tage, wobei die Kontagiosität wahrscheinlich schon vor dem Auftreten von Symptomen beginnt und über 2-3 Wochen nach initialer Infektion anhält.

Eine Infektion mit Herpes-simplex-Viren kann als Primär- und als Re-Infektion ablaufen, wobei die Erstinfektion normalerweise ohne Folgeschäden abläuft.

Cave. Bei Neugeborenen können Infektionen mit Herpes-simplex-Viren lebensbedrohlich verlaufen und müssen daher unverzüglich therapiert werden.

Das Molluscum-contagiosum-Virus gehört zu den Poxviren. Eine Ansteckung erfolgt direkt von Mensch zu Mensch oder über erregertragende Instrumente.

\section{Symptomatik}

\section{- Adenovirusinfektionen}

Die KCE ist die schwerste Form einer Adenovirusinfektion der Augenoberfläche. Die Patienten beschreiben häufig ein Fremdkörpergefühl, welches nasal beginnt und von objektiven Symptomen wie Chemosis, Bindehautrötung, Lidschwellung, Tränen und Visusverschlechterungen begleitet wird. Typisch ist eine präaurikuläre oder zervikale Lymphknotenschwellung sowie eine Schwellung der Plica semilunaris (Abb. 2b) und der Karunkel des Auges.

Teilweise zeigen sich Pseudomembranen der Bindehaut. Typisch ist eine akute Beteiligung der Hornhaut, wobei zunächst eine Keratitis punctata superficialis bestehen kann. In der chronischen Phase bilden sich korneale Immunkomplexe als sog. Nummuli aus, die das Sehvermögen langfristig reduzieren können.

Der Schweregrad der Erkrankung variiert stark von subklinisch bis hin zu schwersten Verläufen mit systemischen Symptomen wie zum Beispiel Gliederschmerzen und allgemeinem Krankheitsgefühl. Die akute Phase dauert ca. 4-6 Wochen, wobei die Nummuli über viele Monate bis Jahre bestehen bleiben können. Ein weiteres Residuum einer KCE kann ein „Trockenes Auge“ sein [7]. 


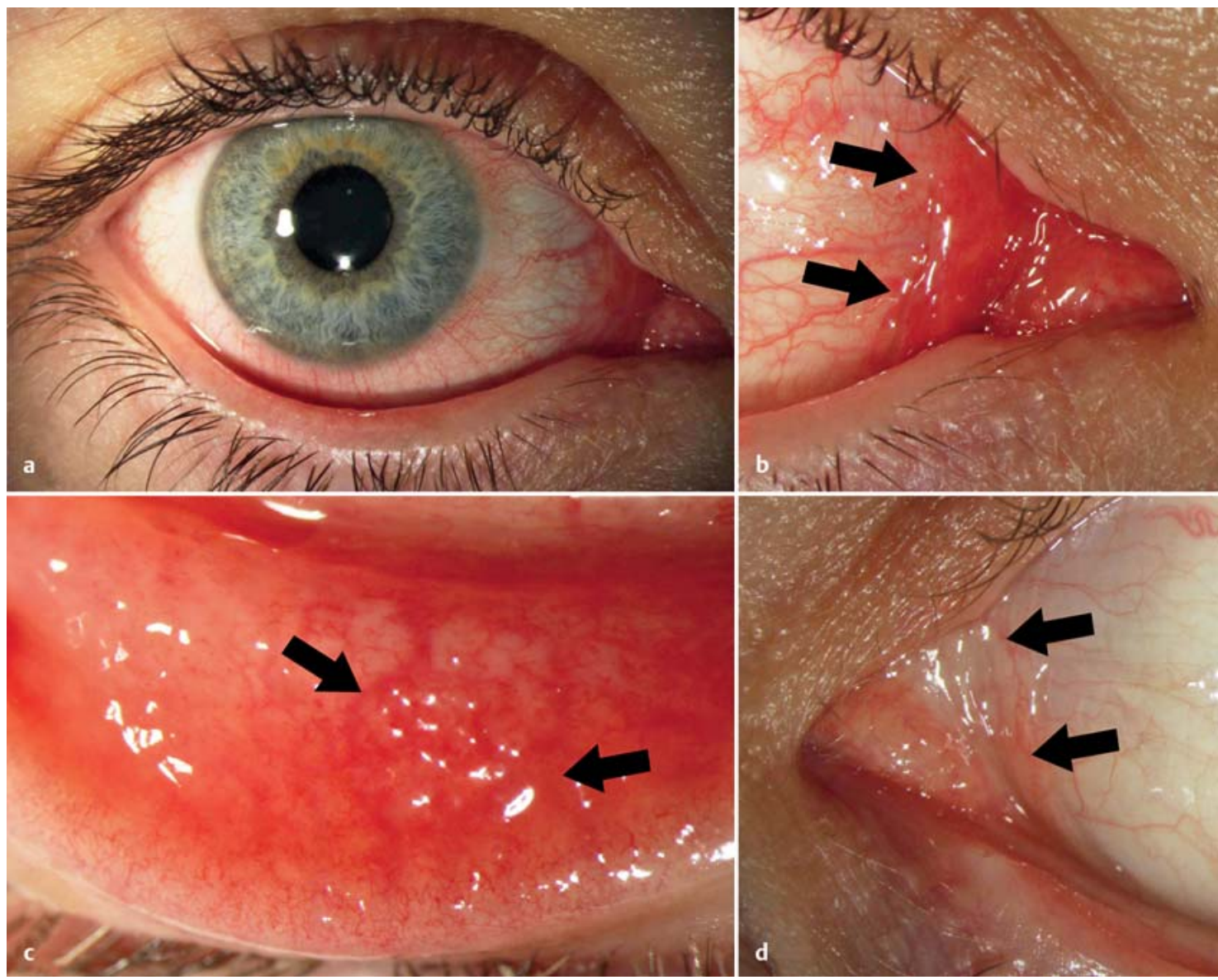

Abb. 2 Keratoconjunctivitis epidemica. a Akute Keratoconjunctivitis epidemica. b Geschwollene Plica semilunaris (Pfeile). c Follikel (Pfeile) in der tarsalen Bindehaut inferior. d Normale Plica semilunaris (Pfeile), kontralaterales Auge.

\section{Checkliste}

Klinische Symptome der Keratoconjunctivitis epidemica (KCE)

- Fremdkörpergefühl

- Chemosis

- Bindehautrötung

- Lidschwellung

- Tränen

- Visusverschlechterung

- Schwellung der Plica semilunaris und der Karunkel

- präaurikuläre oder zervikale Lymphknotenschwellung

- Pseudomembranen der Bindehaut

- akute Beteiligung der Hornhaut

- korneale Immunkomplexe (in der chronischen Phase)

\section{- Herpes-simplex-Virus-Infektionen}

Die primäre HSV-Infektion verläuft oft, aber nicht immer, mit typischen Lid- oder Hornhautveränderungen. Die Bindehaut zeigt häufig eine Rötung und eine folli- kuläre Reaktion, die in ihrer Ausprägung stark variieren kann.

Der klinische Verlauf dauert normalerweise 2 Wochen, wobei die meisten Patienten nur unilateral betroffen sind. Andere Symptome sind Fremdkörpergefühl, Brennen und Tränen.

\section{Molluscum-contagiosum-Virus}

Die Virusinfektion führt zur Entstehung von Dellwarzen, vor allem im Kopf- und Halsbereich. Immunsupprimierte Patienten können über 100 dieser Hautveränderungen aufweisen. Die leicht erhabenen weißlichen Papeln zeigen oft eine zentrale Vertiefung, die mit einem exprimierbaren schwammigen Material gefüllt ist. Dieses Material ist hochinfektiös und kann leicht in die Bindehaut gelangen, wo es eine follikuläre chronische Konjunktivitis auslöst. 


\section{Diagnostik}

Die Diagnose der KCE wird in den meisten Fällen klinisch gestellt. Aktuell gibt es außerdem AdenovirenSchnelltests, die jedoch bislang - zumeist aus Kostengründen - nur in unklaren oder besonders schweren Fällen eingesetzt werden. Die Sensitivität und Spezifität dieser Schnelltests liegen bei bis zu 90\% bzw. 96\%.

Der direkte Nachweis von Adenoviren im Konjunktivalabstrich ist meldepflichtig.

Nach § 7 (1) IfSG hat der positive Nachweis einer Adenovirusinfektion der Bindehaut namentlich innerhalb von 24 Stunden nach erlangter Kenntnis an das zuständige Gesundheitsamt zu erfolgen, wobei nach Information des Robert Koch-Instituts in einigen Bundesländern schon das klinische Bild der KCE meldepflichtig ist (detaillierte Informationen zur KCE unter www.rki.de).

Die Diagnose der HSV-Konjunktivitis erfolgt meistens klinisch aufgrund gleichzeitig bestehender Hautveränderungen (Bläschen) oder aufgrund typischer Hornhautveränderungen (Keratitis superficialis, dendritiformer Epithelschäden, etc.). In atypischen Fällen und bei Neugeborenen kann eine HSV-PCR notwendig sein.

Die Diagnose der Molluscum-contagiosum-VirusInfektion wird klinisch durch das typische Aussehen der Dellwarzen gestellt. Vor allem die Lidränder sollten mit der Spaltlampe sorgfältig untersucht werden.

\section{Therapie}

\section{Keratoconjunctivitis epidemica (KCE)}

Eine sichere und kausale Therapie der KCE ist bislang nicht vorhanden. Empfohlen werden die Applikation von Tränenersatzmitteln und in Einzelfällen die Gabe von topischen Antibiotika zur Behandlung von bakteriellen Superinfektionen. In einzelnen Studien wurden vorteilhafte Effekte und eine Reduktion der Viruslast durch die Applikation von Ganciclovir oder PovidonJod gezeigt [8].

Eine Gabe von Steroiden in der akuten Phase wird jedoch nicht empfohlen, da klinische Studien zwar eine anfängliche Reduktion der Symptome zeigten, sie letztlich jedoch zu einer Verlängerung der Gesamtheilungszeit führte [9]. Für die Behandlung der chro-

\section{Checkliste}

Hygienemaßnahmen bei Keratoconjunctivitis epidemica (KCE)

- Händedesinfektion mit viruziden Desinfektionsmitteln

- Desinfektion aller Gegenstände, mit denen der Patient in Berührung gekommen ist

- Meidung von Menschenmengen in der kontagiösen Phase

- Arbeitsunfähigkeitsbescheinigung für den Zeitraum der Infektiosität (mind. 2-3 Wochen)

- räumliche Separation wartender Patienten mit KCE-Verdacht

nischen Hornhautveränderungen wird auf den Beitrag zur infektiösen Keratitis verwiesen.

In Ergänzung der Therapie ist vor allem die Einhaltung hygienischer Maßnahmen zur Ansteckungsprophylaxe von vordringlicher Bedeutung. So ist insbesondere die Händedesinfektion und die Desinfektion aller mit dem Patienten in Berührung gekommenen Gegenstände (Spaltlampe, Instrumente, Türgriffe, Stühle, etc.) mit viruziden Desinfektionsmitteln oder mit thermischen Desinfektionsverfahren $\left(93^{\circ} \mathrm{C}, 5 \mathrm{~min}\right)$ sehr wichtig. Wartende Patienten mit Verdacht auf KCE sollten zudem von anderen Patienten räumlich separiert werden.

KCE-Erkrankte sollten über die hohe Infektiosität der Erkrankung informiert werden. Sie sollten in der kontagiösen Phase Menschenmengen meiden und über den Zeitraum der Infektiosität (also mindestens über 2-3 Wochen) eine Arbeitsunfähigkeitsbescheinigung erhalten [9].

\section{- HSV-Konjunktivitis}

Die Behandlung der HSV-Konjunktivitis erfolgt mit Aciclovir oder Gancliclovir als Salbe oder Gel mehrfach täglich appliziert. Die Dauer der Behandlung sollte mindestens 14 Tage betragen, ist jedoch vom klinischen Verlauf und eventueller begleitender Infektionen der Hornhaut oder intraokulärer Strukturen abhängig. Von einer Applikation von Steroiden zur Behandlung der HSV-Konjunktivitis wird abgeraten. 


\section{Molluscum-contagiosum-Infektionen}

Die Behandlung einer Molluscum-contagiosum-Infektion sollte vor allem bei immunsupprimierten Patienten erfolgen. Ansonsten kann bei unauffälligem Augenbefund ein Spontanverlauf abgewartet werden. Behandlungsmöglichkeiten umfassen eine Kryotherapie, eine Kürettage oder eine Exzision. Rückfälle sind möglich.

\section{Mykotische Konjunktivitis}

\section{Zusammenfassung}

Die mykotischen Konjunktivitiden sind in unseren Breiten selten, können jedoch sehr vielgestaltig erscheinen. Eine Beteiligung der regionalen Lymphknoten ist möglich. Die Diagnose wird meist spät durch Biopsie und histopathologische Untersuchung gestellt, weshalb die Erkrankung auch als deutlich unterdiagnostiziert gilt.

\section{Epidemiologie und Ätiologie}

Exakte epidemiologische Daten zur Pilz-Konjunktivitis fehlen, sie wird jedoch in unseren Breiten als selten angenommen. Ätiologisch spielen Mikrotraumata insbesondere im landwirtschaftlichen Kontext eine wesentliche Rolle. Auch nach längerer topischer Steroidtherapie kann es zu einer Pilzinfektion der Bindehaut kommen. Ebenso wird ein gehäuftes Auftreten bei immunsupprimierten Patienten beobachtet.

\section{Symptomatik}

Symptomatisch ist die mykotische Konjunktivitis den anderen infektiösen Bindehautentzündungen sehr ähnlich, nicht zuletzt, da häufig eine Mischinfektion vorliegt. Typisch für eine Pilzinfektion ist der subakute bis chronische schwere Verlauf bei entsprechender Anamnese (landwirtschaftlicher Kontext, topische Steroid-Langzeittherapie, Immunsuppression).

\section{Diagnostik}

An erster Stelle des diagnostischen Vorgehens steht, wie bei allen Konjunktivitiden, die genaue spaltlampenbiomikroskopische Inspektion der vollständigen
Bindehaut unter Ektropionieren von Ober- und Unterlidern. Da sich das klinische Bild sehr variabel gestaltet, wird die Diagnose einer mykotischen Konjunktivitis in der Regel durch Bindehautbiopsie und histopathologische Untersuchung gestellt. Klinisch wie histopathologisch hat sich folgende Einteilung etabliert:

- oberflächliche mykotische Konjunktivitis

- proliferierende mykotische Konjunktivitis

- tiefe granulomatöse mykotische Konjunktivitis

Oberflächliche mykotische Konjunktivitis. Hier sind überwiegend die epithelialen und subepithelialen Schichten der Bindehaut betroffen. Zu den häufigsten Erregern zählen

- Candida albicans,

- Pityrosporum ovale und

- Dermatophyten wie Mikrosporum oder Trichophyton.

Die seltene konjunktivale Candidainfektion imponiert klinisch als pseudomembranöse Konjunktivitis, häufig im Rahmen einer generalisierten Moniliasis (Soor). Im fortgeschrittenen Stadium kann es zu Bindehautschrumpfungen mit Symblepharonbildung und vaskularisierter Hornhauttrübung kommen.

Das Pityrosporum ovale tritt häufig zusammen mit pathogenen Staphylokokken im Rahmen einer seborrhoischen (Blepharo-)Konjunktivitis auf.

Proliferierende mykotische Konjunktivitis. Papillomatöse bis pustulöse Veränderungen zeigen sich bei der

- Blastomykose,

- Rhinosporidiose,

- Aspergillose,

- Coccidioidose und

- Paracoccidioidose.

Die Blastomykose imponiert klinisch als purulente, ulzerierende Läsion, umgeben von Granulationsgewebe, und tritt meist im Fornix conjunctivae auf, seltener im Bereich der bulbären Bindehaut, wo sie einem BitotFleck zum Verwechseln ähnlich sein kann. Zugleich kommt es zu einer regionalen Lymphadenopathie. Histopathologisch findet sich eine granulomatöse Entzündung mit großen mononukleären und multinukleären Zellen.

Die vorwiegend in den Tropen auftretende Rhinosporidiose ist in der Regel unilateral ohne regionale Lymphadenopathie. Sie imponiert klinisch als flaches, teils polypöses, schmerzloses Granulom der Conjunc- 
tiva bulbi et tarsi des Unterlides, häufig einem aufbrechenden Chalazion ähnlich. Histopathologisch zeigen sich zahlreiche Zysten unter einem proliferierten Epithel eingebettet in fibrozelluläres Gewebe, die teils leer, teils mit Rhinosporen gefüllt sind und sich bei spontaner Ruptur in den Bindehautsack entleeren können. Auch hier wird die Diagnose in der Regel durch die histopathologische Untersuchung gestellt.

Tiefe granulomatöse mykotische Konjunktivitis. Subepithelial oder sogar subkonjunktival finden sich knötchenförmige, granulomatöse Entzündungsherde, umgeben von proliferierendem Bindegewebe, die ulzerieren und eitrig einschmelzen können. Häufigster Erreger ist das Sporotrichum schenckii, ein Pflanzensaprophyt, der durch Mikrotraumen die Bindehaut infizieren kann. Typisch für eine Sporotrichose ist die Assoziation mit einer Lymphangitis und Lymphadenopathie, insbesondere der präaurikulären Lymphknoten.

\section{Zielgerichtete Diagnostik}

Diagnostisches Vorgehen bei mykotischer Konjunktivitis

- Anamnese (landwirtschaftlicher Kontext, topische Steroid-Langzeittherapie, Immunsuppression)

- spaltlampenbiomikroskopische Inspektion der vollständigen Bindehaut

- Bindehautbiopsie und histopathologische Untersuchung

\section{Therapie}

Nach Biopsie und Diagnosestellung wird lokal und ggf. systemisch eine antimykotische Therapie eingeleitet. Die fungizide Wirksubstanz richtet sich, sofern möglich, nach dem identifizierten Erreger, z.B.

- Fluconazol $0,2 \%$

- Voriconazol $1 \%$ oder

- Amphotericin B 0,5\% Augentropfen.

\section{Perspektiven}

In Zukunft könnte die In-vivo-Konfokalmikroskopie der Augenoberfläche Bedeutung in der Diagnostik der mykotischen Konjunktivitis erlangen.

\section{Parasiten- und Raupenhaar- Konjunktivitis}

\section{Zusammenfassung}

Die parasitären Konjunktivitiden gehören zu den Tropenerkrankungen und sind in unseren Breiten sehr selten. Die meist granulomatösen Entzündungen sind häufig visusbedrohend und sollen hier nur im Überblick angerissen werden.

\section{Übersicht}

Erreger parasitärer Konjunktivitiden

- Protozoen: Leishmaniose

- Plathelminthen: Schistosomiasis, Echinococcose, Zystizerkose

- Nematoden: Filariasis, Onchozerkose

- Fliegen (Eier und Larven)

- Raupen (Raupenhaar-Konjunktivitis)

\section{Epidemiologie und Ätiologie}

Es liegen keine epidemiologischen Daten zur ParasitenKonjunktivitis in unseren Breiten vor. Aber im Zuge der Globalisierung und des Tropentourismus ist mit einer Zunahme ihrer Inzidenz zu rechnen.

In der Regel handelt es sich um eine granulomatöse Entzündung durch Protozoen (Leishmaniose), Plathelminthen (Schistosomiasis, Echinococcose, Zystizerkose) oder Nematoden (Filariasis, Onchozerkose).

Auch Eier und Larven von Fliegen können eine granulomatöse Konjunktivitis verursachen (okuläre Myiasis). Die Härchen von Raupen rufen eine schwere Fremdkörperreaktion mit zahlreichen granulomatösen Entzündungsherden hervor (Raupenhaar-Konjunktivitis). Penetrieren die Raupenhaare den Bulbus, kann es zu einer schweren granulomatösen Endophthalmitis kommen.

\section{Symptomatik}

Die Beschwerden wie auch das klinische Bild sind sehr variabel. Häufig dominieren Juckreiz und Brennen der Augen, teilweise ausgelöst durch die Bewegung der Parasiten. 


\section{Checkliste}

Diagnostik bei parasitärer Konjunktivitis

- eingehende Anamneseerhebung, insbesondere Reiseund Haustieranamnese

- primäre Inspektion der Bindehaut

- spaltlampenbiomikroskopische Inspektion der vollständigen Konjunktiva

- Biopsie und histopathologische Untersuchung

- hilfreich: In-vivo-Konfokalmikroskopie der Augenoberfläche

\section{Diagnostik}

Der eingehenden Anamneseerhebung (insbesondere Reise- und Haustieranamnese) folgt die spaltlampenbiomikroskopische Inspektion der vollständigen Konjunktiva unter Ektropionieren von Ober- und Unterlidern. Zum Teil lassen sich die Parasiten direkt visualisieren, zum Teil zeigen sich Bindehautgranulome. Auch eine primäre Inspektion der Bindehaut mit bloßem Auge ohne weitere Hilfsmittel kann nützlich sein, da beispielsweise der bis zu $7 \mathrm{~cm}$ lange Fadenwurm Loa-Loa vor dem Licht der Spaltlampe flüchtet. Die In-vivo-Konfokalmikroskopie der Augenoberfläche kann ebenso hilfreich sein vor entsprechender Biopsie und histopathologischer Untersuchung. Hier imponieren meist nekrotische Reste von Parasiten umgeben von Riesenzellen und vielen Eosinophilen (SplendoreHoeppli-Reaktion).

Cave. Bei Vorliegen einer parasitären Konjunktivitis ist eine komplette ophthalmologische Untersuchung inklusive Hornhaut- und Fundusuntersuchung in Mydriasis obligatorisch, um eine okuläre Beteiligung oder Sekundärveränderungen (wie neovaskuläre Hornhauttrübungen) auszuschließen [10].

\section{Therapie}

Die Behandlung der parasitären Konjunktivitis ist sehr unterschiedlich und reicht von der einfachen Entfernung der Parasiten mittels Pinzette unter lokaler Betäubung bis hin zur chirurgischen Exzision. Darüber hinaus können desinfizierende, antiparasitäre wie auch antiinflammatorische Medikationen lokal und ggf. systemisch eingesetzt werden.

\section{Quellenangaben}

1 Smith AF, Waycaster C. Estimate of the direct and indirect annual cost of bacterial conjunctivitis in the United States. BMC Ophthalmol 2009; 9: 13

2 Gille G, Klapp C. Chlamydia trachomatis infections in teenagers. Hautarzt 2007; 58: 31 - 37

3 Soukiasian SH, Baum J. Bacterial Conjunctivitis. In: Krachmer JH, Mannis MJ, Holland EJ. Cornea. Philadelphia: Elsevier Mosby; 2005: 615-628

4 Meyer T. [Modern diagnosis of Chlamydia trachomatis infections]. Hautarzt 2007; 58: 24-30

5 Messmer EM. Bakterielle Konjunktivitis - Update zu Diagnose und Therapie. Klin Monatsbl Augenheilkd 2012; 228: 529-533

6 Meyer-Rüsenberg B, Loderstädt U, Richard G et al. Keratokonjunktivitis epidemica: Infektionslage und aktuelle Hinweise zur Prophylaxe und Therapie. Dtsch Ärzteblatt Int 2011; 108: 475-480

7 Trousdale MD, Goldschmidt PL, Nóbrega R. Activity of ganciclovir against human adenovirus type- 5 infection in cell culture and cotton rat eyes. Cornea 1994; 13: 435-439

8 Pelletier JS, Stewart K, Trattler W et al. A combination povidoneiodine $0.4 \%$ /dexamethasone $0.1 \%$ ophthalmic suspension in the treatment of adenoviral conjunctivitis. Adv Ther 2009; 26:

$776-783$

9 Isenberg SJ, Apt L, Valenton M et al. A controlled trial of povidoneiodine to treat infectious conjunctivitis in children. Am J Ophthalmol 2002; 134: 681-688

10 Völcker HE, Naumann GOH. Konjunktiva. In: Naumann GOH. Pathologie des Auges. Berlin, Heidelberg, New York: Springer; 1980: $240-312$

Interessenkonflikt: Die Autoren bestätigen, dass kein Interessenkonflikt vorliegt.

\section{Dieser Artikel erschien in den \\ Klinischen Monatsblättern für Augenheilkunde \\ (DOI 10.1055/s-0032-1325087).}

\section{Korrespondenzadresse}

PD Dr. Philipp Steven

Zentrum für Augenheilkunde

Kerpener Str. 62

50937 Köln

E-Mail: philipp.steven@uk-koeln.de 


\section{CME.thieme.de}

\section{CME-Fragen}

\section{CME-Teilnahme}

- Viel Erfolg bei Ihrer CME-Teilnahme unter http://cme.thieme.de

- Bitte informieren Sie sich über die genaue Gültigkeitsdauer unter http://cme.thieme.de

- Sollten Sie Fragen zur Online-Teilnahme haben, unter http://cme.thieme.de/hilfe finden Sie eine ausführliche Anleitung.

\section{1}

Welche Aussage ist richtig?
A Die Bindehaut ist grundsätzlich als sterile Oberfläche anzusehen.
B Die Bindehaut besitzt eine sog. kommensale Flora.
C Das Immunsystem der Bindehaut besteht ausschließlich aus diffusem und organisiertem konjunktivaassoziiertem lymphatischem Gewebe.
D Propionibakterien gehören zu den gefährlichsten Keimen an der Augenoberfläche.
E Immunglobuline haben keinen Einfluss auf die Schleimhautabwehr.

\section{2}

Durch welchen der folgenden Risikofaktoren wird eine bakterielle Konjunktivitis nicht begünstigt?
A Trockenes Auge.
B Lagophthalmus.
C Bestrahlung der Augenoberfläche.
D Das Alter.
E Okuläre Graft-versus-Host-Erkrankung.

\section{3}

Welcher sexuell übertragbare Erreger ist besonders häufig für eine Bindehautentzündung verantwortlich?
A Chlamydia trachomatis.
B Treponema pallidum.
C Gardnerella vaginalis.
D Humane Papillomaviren (HPV).
E Trichomonas vaginalis.

4

Zur hyperakuten Form der bakteriellen Konjunktivitis zählt typischerweise die
A Chlamydienkonjunktivitis.
B Actinomycetenkonjunktivitis.
C Gonokokkenkonjunktivitis (Gonoblenorrhoe).
D Staphylokokkenkonjunktivitis.
E Streptokokkenkonjunktivitis.

\section{5}

Welche Aussage trifft zu?
A Bei jedem Patienten mit Bindehautentzündung muss zwingend ein Abstrich entnommen werden.
B Konjunktivitiden weisen eine nur sehr niedrige Spontanheilungsrate auf.
C Papillen weisen immer auf eine Allergie hin und sind als pathognomonisch hierfür zu werten.
D Follikel sind beweisend für eine Chlamydieninfektion.
E Chlamydien können mittlerweile über eine PCR-Untersuchung nachgewiesen werden.

\section{6}

Adenoviren können verschiedene Erkrankungen der Augenoberfläche auslösen. Dazu gehört nicht:
A pharyngokonjunktivales Fieber (PKF).
B Keratoconjunctivitis epidemica (KCE).
C akute nicht spezifische Konjunktivitis.
D chronische Keratokonjunktivitis.
E hyperakute eitrige Konjunktivitis. 


\section{CME-Fragen}

Bindehautinfektionen

\section{7}

Welches diagnostische Zeichen ist typisch für eine Molluscumcontagiosum-Infektion?
A Riesenpapillen insbesondere unter dem Oberlid.

B Chronische follikuläre Konjunktivitis.

C Dendritiforme Epithelschäden der Hornhaut.

D Eitriger Reflux aus den Tränenpünktchen.

E Reduktion der Hornhautsensibilität.

\section{8}

Was gehört nicht zu den typischen Symptomen einer Keratoconjunctivitis epidemica (KCE)?
A Erhöhter intraokulärer Druck.
B Follikel der Bindehaut.
C Seröses Sekret.
D Geschwollene Plica semilunaris.
E Geschwollene ipsilaterale präaurikuläre und/oder zervikale Lymphknoten.

9

Welche Aussage trifft zu?

Pilzkonjunktivitiden sind
A in nordeuropäischen Regionen eher selten.
B klinisch leicht zu diagnostizieren.
C zumeist nicht behandlungsbedürftig.
D ausschließlich auf das Bindehautepithel beschränkt.
E immer durch Dermatophyten verursacht.

\section{0}

Bei welcher der folgenden Erkrankungen tritt eine parasitäre Konjunktivitis nicht auf?
A Leishmaniose.
B Schistosomiasis.
C Filariasis.
D Onchozerkose.
E Blastomykose. 